\title{
Variation in risk of second primary cancer
}

\author{
Marcy Winget PhD, Yutaka Yasui PhD
}

See related research article by Nielsen and colleagues on page E57 and at www.cmaj.ca/lookup/doi/10.1503/cmaj.110167

$\mathrm{S}$ econd primary cancer is a major source of concern among cancer survivors, their families and the health care professionals providing follow-up care. Its risk is determined in a complex interaction between treatment of the first cancer, the genetic profile of the survivor, and environmental and behavioural factors. For example, exposure to specific treatments for cancer in childhood has been linked to an increased risk of certain second primary cancers in adulthood. ${ }^{1,2}$ This increased risk has resulted in the development of specific evidence-based follow-up guidelines by the Children's Oncology Group. ${ }^{1}$

Certain germline mutations of the $p 53$ tumour suppressor gene, responsible for most instances of Li-Fraumeni syndrome, elevate a person's risk of certain cancers, any pair of which could appear as the first and second primary cancers. ${ }^{3}$ Possibly most important from the perspective of prevention, risks of certain cancers can be jointly elevated by specific environmental and behavioural factors. For instance, a population-based nested case-control study involving breast cancer survivors showed that obesity, alcohol intake and smoking increased the risk of a second breast cancer appreciably. ${ }^{4}$

Not all second cancers are attributable to known risk factors, however. It is therefore important to study the associations between first and second primary cancers in an effort to better understand their cause and develop informed recommendations for follow-up care.

In a related article in CMAJ, Nielsen and colleagues present their findings from a nationwide nested case-control study in Denmark that assessed the risk of second primary cancer. ${ }^{5}$ The study has a number of methodologic strengths: the authors identified a large number of patients with cancer from a high-quality national registry over a recent 28-year period (1980-2007); each patient was matched (by sex, birth year, calendar period at risk and age at diagnosis of the cancer) with up to five randomly selected controls who did not have the examined cancer at the time of diagnosis; they performed sensitivity analyses that included cancers diagnosed only 1,2, 5 and
10 years after the first cancer diagnosis, to minimize the potential of erroneously including recurrent cancer as a new primary cancer; and they used state-of-the-art statistical methods that accounted for multiple comparisons of many types of first and second primary cancers.

Well-designed, well-conducted studies such as the one by Nielsen and colleagues can provide important data to inform causal mechanisms and follow-up care of cancer survivors. Specifically, the detailed cancer-pair-specific analyses of Nielsen and colleagues are valuable for exploring causes such as shared genetic predisposition and for evaluating or modifying guidelines for follow-up care (although the analyses did not consider treatment factors of the first primary cancer).

Two key issues exist in translating the findings on the risk of second primary cancer, such as those of Nielsen and coauthors, into clinical implications on the frequency and type of follow-up care. The first issue is the heterogeneity in risk of second primary cancer across pairs of first and second cancers. Nielsen and colleagues found that the risk of a second primary cancer depended greatly on the types of the first and second cancers; heterogeneity in risk was substantial across cancer types, regardless of whether the second cancer was the same type as the first. Their study provides evidence of the high degree of heterogeneity (even though they attempted to synthesize the data using fixedeffect meta-analysis techniques) and thus the need for separate evaluation and discussion of

\section{KEY POINTS}

- Second primary cancer is a major concern for cancer survivors, their families and the health care professionals providing follow-up care.

- A nationwide study of the associations between first and second primary cancers showed more than a twofold risk overall of a second primary cancer of the same type as the first.

- However, the high degree of heterogeneity in risk observed across cancer types indicates the need for separate evaluation and discussion of risk by specific type of first-second cancer pair rather than overall risk.

- The absolute risk of each type of second primary cancer should influence clinical decisions around the frequency and type of follow-up care. 
risk by specific type of first-second cancer pair rather than overall risk.

The second issue is the notion of absolute risk, which, in general, critically dictates health care and public health practice. Absolute risk cannot be estimated in a matched case-control study, but it is what dictates priorities in the follow-up care of cancer survivors. As an example, Nielsen and colleagues report a hazard ratio of 17.8 for a second sarcoma after an initial sarcoma, the largest hazard ratio in the study. This does not imply that the follow-up care of sarcoma survivors should focus only on potential second primary sarcoma. The five-year absolute risk to sarcoma survivors of acquiring breast cancer, for example, was higher than that of a second primary sarcoma, even though the hazard ratio (relative to the general population) for breast cancer as a second primary cancer was only 1.46. Because of the higher absolute risk of breast cancer than of sarcoma, follow-up care targeted to identifying breast cancer is as important as that for identifying a second sarcoma, despite the large difference in the hazard ratios. A further nuance applies to sarcoma survivors in view of the Li-Fraumeni syndrome, ${ }^{3}$ which elevates the risk of several cancers, including sarcoma and breast cancer. Sarcoma survivors with certain family histories of cancer may be carriers of the genetic mutations; follow-up care of such patients is complex, precisely because the absolute risk of breast or other types of cancer can be high.
In summary, Nielsen and coauthors estimated hazard ratios of second primary cancer by type or site of the first and second cancers in a sound epidemiologic study. Their well-conducted study provides valuable results. Caution must be exercised, however, in interpreting the findings for implications for clinical practice, in view of the substantial heterogeneity in risk of second primary cancer across cancer types and the absolute risk of second primary cancers.

\section{References}

1. Landier W, Bhatia S, Eshelman DA, et al. Development of riskbased guidelines for pediatric cancer survivors: the Children's Oncology Group Long-Term Follow-up Guidelines from the Children's Oncology Group Late Effects Committee and Nursing Discipline. J Clin Oncol 2004;22:4979-90.

2. Neglia JP, Friedman DL, Yasui Y, et al. Second malignant neoplasms in five-year survivors of childhood cancer: childhood cancer survivor study. J Natl Cancer Inst 2001;93:618-29.

3. Li FP, Fraumeni JF Jr. Soft-tissue sarcomas, breast cancer, and other neoplasms. A familial syndrome? Ann Intern Med 1969;71: 747-52.

4. Li CI, Daling JR, Porter PL, et al. Relationship between potentially modifiable lifestyle factors and risk of second primary contralateral breast cancer among women diagnosed with estrogen receptor-positive invasive breast cancer. J Clin Oncol 2009;27: 5312-8.

5. Nielsen S, Nordestgaard B, Bojesen S. Associations between first and second primary cancers: a population-based study. CMAJ 2012;184:E57-69.

Affiliations: Marcy Winget is with Cancer Care, Alberta Health Services and the School of Public Health, University of Alberta, Edmonton, Alta. Yutaka Yasui is with the School of Public Health, University of Alberta, Edmonton, Alta.

Contributors: Both authors contributed to drafting and revising the manuscript and approved the final version submitted for publication. 